\title{
Nondestructive monitoring of aircraft composites using terahertz radiation
}

\author{
Nikolay S. Balbekin ${ }^{a}$, Evgenii V. Novoselov ${ }^{b}$, Pavel V. Pavlov ${ }^{c}$, Victor G. Bespalov ${ }^{a}$, \\ Nikolay V. Petrov ${ }^{a}$ \\ ${ }^{a}$ Department of photonics and optical informatics, ITMO University, Kadetskaya Linia 3, \\ St. Petersburg, Russia, 199004. \\ ${ }^{b}$ Chalmers Univ. of Technology, Chalmersplatsen 4, Gothenburg, Sweden, 41296. \\ ${ }^{c}$ Military Aviation Engineering University, Starih Bolshevikov st. 54-a, Voronezh, Russia, \\ 394064.
}

\begin{abstract}
In this paper we consider using the terahertz (THz) time domain spectroscopy (TDS) for non destructive testing and determining the chemical composition of the vanes and rotor-blade spars. A versatile terahertz spectrometer for reflection and transmission has been used for experiments. We consider the features of measured terahertz signal in temporal and spectral domains during propagation through and reflecting from various defects in investigated objects, such as voids and foliation. We discuss requirements are applicable to the setup and are necessary to produce an image of these defects, such as signal-to-noise ratio and a method for registration $\mathrm{THz}$ radiation. Obtained results indicated the prospects of the THz TDS method for the inspection of defects and determination of the particularities of chemical composition of aircraft parts.
\end{abstract}

Keywords: Terahertz radiation, terahertz spectroscopy, terahertz imaging, nondestructive testing, aircraft.

\section{INTRODUCTION}

The qualitatively new technologies are implemented in a global aircraft industry to ensure not only flight safety but also maximum efficiency of aircraft operation. Substantially it is achieved by increasing use of composite materials for aircraft. The composition and therefore the properties of these materials may varied depending on desired values of strength, heat resistance, modulus, abrasion resistance, and moreover differ in their magnetic, dielectric, radio and other properties. Glass fiber, epoxy resin and carbon fiber composite materials are increasingly used as structural airframe elements, due to their high strength to weight ratio, improved aerodynamic performance, reliability, and reduced corrosiveness compare to other construction materials. However, composite structure modifications during the exploitation are possible due to the strong thermal influence (e.g., from turbines) or various material strains due to external influences (such as unsuccessful plane landing or bullet hits). Also material deterioration is possible caused by a prolonged operation period. The widespread use of composites requires a new approaches both for aircraft design and manufacture, and maintenance and repair services including the high-quality diagnostics. The most common methods for non-destructive testing are ultrasonic techniques, ${ }^{1,2}$ thermography ${ }^{3,4,5}$ and shearography, ${ }^{4,6}$ but all of them have some disadvantages as expensiveness, insufficient sensitivity, a problem with the detection of subsurface defects and suitability not for all kinds of defects. ${ }^{7}$

During the past few years $\mathrm{THz}$ spectroscopy has been applied as a technic for non-contact composite material diagnostics. THz radiation is located between infrared and microwave bands of electromagnetic spectrum. High transparency of smoke, clothing, paper, wood, plastic, ceramic surfaces and other materials for $\mathrm{THz}$ radiation provides a great potential for intravision with high signal to noise ratio. THz radiation is non-ionizing unlike Xrays and has less scattering and greater penetration depth in comparison with optical and infrared radiation. By use of the electro-optical detection in THz TDS it is possible to register real part of THz field complex amplitude, that can provide us information both on amplitude and phase radiation and yield to fuller measurement of optical

E-mail: NBalbekin@niuitmo.ru

Saratov Fall Meeting 2014: Optical Technologies in Biophysics and Medicine XVI; Laser Physics and Photonics XVI; and Computational Biophysics, E. A. Genina, V. L. Derbov, K. V. Larin, D. E. Postnov, V. V. Tuchin, Eds., Proc. of SPIE Vol. 9448, 94482D · C 2015 SPIE · CCC code: 1605-7422/15/\$18 · doi: 10.1117/12.2180021

Proc. of SPIE Vol. 9448 94482D-1 
characteristics. Several papers describing the scattering of $\mathrm{THz}$ radiation in composites $8,9,10,11$ and modeling of their dielectric properties using the effective medium theory $(\mathrm{EMT})^{8,9,10}$ were published. Since the composite is a nonhomogeneous solid material consisting of matrix (binder element) and the reinforcing element, one can determine using EMT its physical properties knowing the properties of components. Application of the $\mathrm{THz}$ imaging methods for monitoring CM showed the possibility of visualization of the spatial distribution of internal defects. ${ }^{11} \mathrm{THz}$ holography and phase retrieval methods ${ }^{12,13,14}$ reveal complete wavefront information both amplitude and phase and provides more complete information about the object under investigation. It is expected to be a more prospective to diagnose composites.

$\mathrm{THz}$ radiation can penetrate into composites and identify defects such as air cavities, delaminations, mechanical damage and thermal damage. In, ${ }^{11}$ results of such thin composite materials defects studies $(\propto 0.1-2.0 \mathrm{~mm})$ using transmission THz TDS were presented. Due to the high THz radiation reflection from relatively polished composite material surface of $30-40 \%$ and both substantial refractive index $(\propto 2)$ and the absorption coefficient, this method has a limitation in application associated with the sample thickness.

In this paper we consider using reflection and transmission THz TDS for non-destructive monitoring of composites. Reflection and transmission of broadband $\mathrm{THz}$ radiation from composite materials were studied using this method (see Fig. 1). Possibility of physical properties determination, various defects identifying and material properties changes were shown due to the harmful effects.

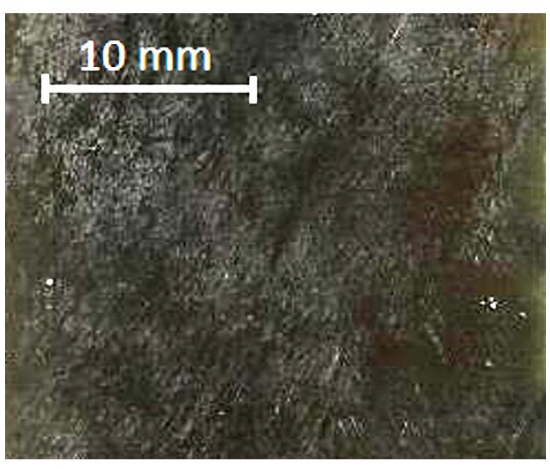

(a)

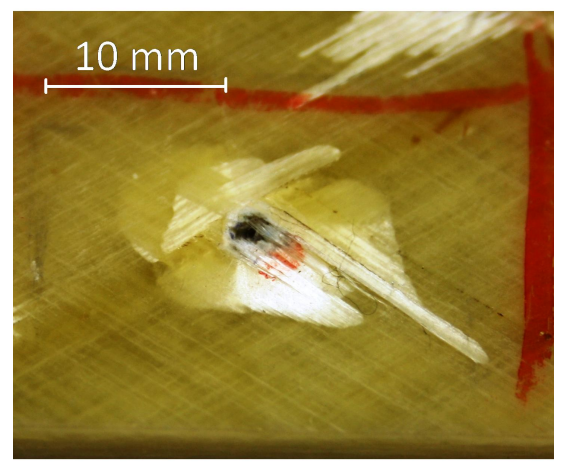

(b)

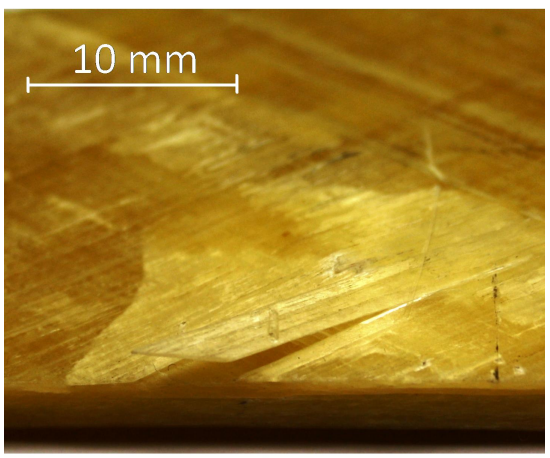

(c)

Figure 1. Studied samples of composite materials. Photograph showing the three samples: (a) burned sample "16a", (b) "17B" sample after mechanical stress, and (c) sample with delamination " $1 \mathrm{~B}$ ".

\section{THE EXPERIMENTAL SETUP}

To generate broadband pulsed THz radiation we used the experimental setup. Photoconductive antenna (undoped InAs) $)^{15,16}$ was placed in a constant magnetic field of $2.4 \mathrm{~T} .{ }^{17}$ Antenna was exposed by radiation of femtosecond laser FL -1 (the active medium - Yb: KYW; $\lambda=1040 \mathrm{~nm}, t_{p}=46 \mathrm{fs}, \nu=70 \mathrm{MHz}, \mathrm{P}=1,2 \mathrm{~W}$ ). It allowed to obtain $\mathrm{THz}$ radiation in conical form with the following parameters: spectral range from 0.05 to $1.6 \mathrm{THz}$, average power $30 \mu \mathrm{W}$, pulse duration $2.7 \mathrm{ps}$. The uppermost energy was distributed between 0.12 and $1.10 \mathrm{THz}$. The scheme of the versatile THz spectrometer is shown on Fig. 2.

$\mathrm{THz}$ radiation from the source passed through a teflon filter to cut off the wavelength range less than 50 microns. Then first PM collimated it and second PM focused it to the sample having a certain amplitude and phase transmittance. The sample was fixed perpendicularly to the optical axis in the focal plane using two axis motorized translation stage. EO detection was carried out using ${ }^{18}$ quarter-wave plate, Wollaston prism, balanced photodetector and lock-in amplifier. Filtered and amplified signal was transmitted to a computer via a digital voltmeter. Spectral resolution during measurements was about $7 \mathrm{GHz}$ for the transmission mode and $15 \mathrm{GHz}$ for reflection mode. ${ }^{19}$ Operation of the setup was realized by means of software environment NI LabVIEW. 


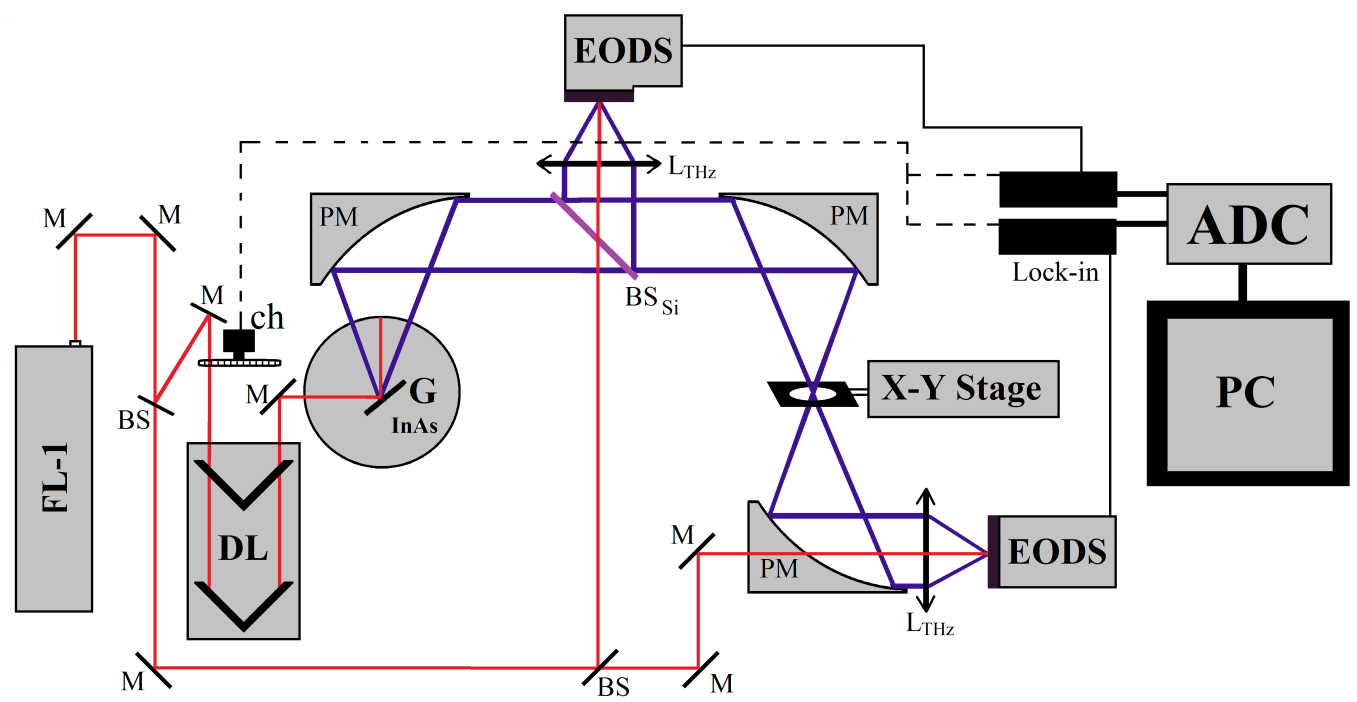

Figure 2. The scheme of versatile THz spectrometer. FL-1 is the femtosecond laser; M are mirrors; BS - beam spleaters; DL - optic delay line; G - THz radiation generator in InAs; PM - parabolic mirrors; ch - chopper; EODS - electro-optical detecting system; lock-in - lock-in amplifier; ADC - analog-digital converter; PC - personal computer.

\section{OBTAINED RESULTS}

Data processing and transmission/reflection spectra preparation were made using a standard procedure applied in THz TDS. Refractive index and dispersion of studied medium was calculated using two complex spectra. ${ }^{20}$ By means of this technique presence of stratification in the composite structure was demonstrate. The change of frequency spectra obtained using FFT in composite structure after heat exposure was shown. Finally, defect visualization due to deformation cause of physical impact was presented.

\subsection{Refraction index and thickness measurement}

Determination of the thickness of the sample using the reflected $\mathrm{THz}$ pulse from the sample was held in two stages. At the first step model samples (steel plates covered with glue with a thickness of $1 \mathrm{~mm}$ ) were studied. We took two kinds of glue for the experiment: epoxy Poxipol, and BF-6. Poxipol is two-component, solvent free, paste-like consistency glue. It is similar in composition to the binder material used for aircraft composite materials synthesis. In contrary to the material, which is used to create composite materials, glue with another parameters was investigated. Glue BF-6 is a simple one-component thermosetting polymerizable glue. Figure 3 shows the investigated samples on a steel substrate.

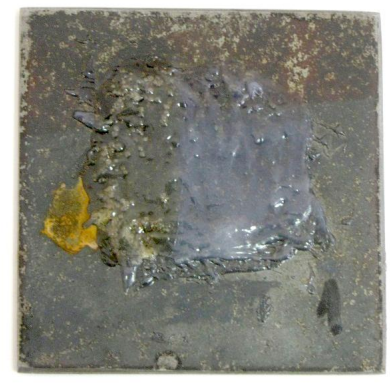

(a)

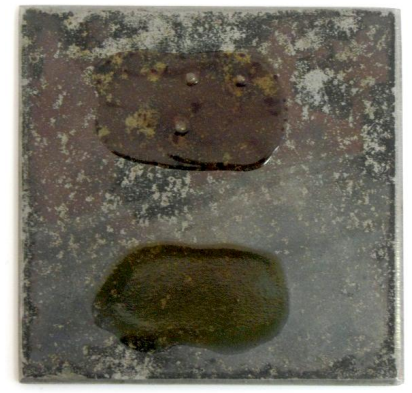

(b)

Figure 3. Studied samples. (a) Epoxy glue Poxipol (on the left side glue BF-6 is applied over Poxipol). (b) Glue BF-6 (on the top side one can see 3 bubbles, which could be assumed as coating defects).

Fig. 4 (a) shows results of $\mathrm{THz}$ pulse reflection from steel surface coated with epoxy glue Poxipol. One can see two distinct reflection from air-glue and glue-steel surfaces. 


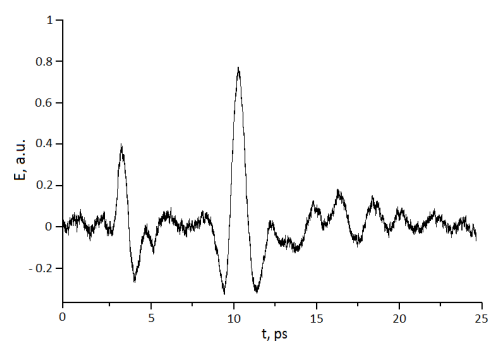

(a)

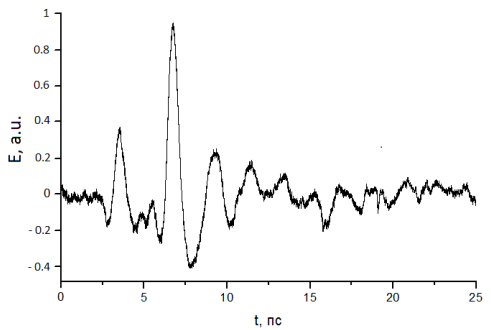

(b)

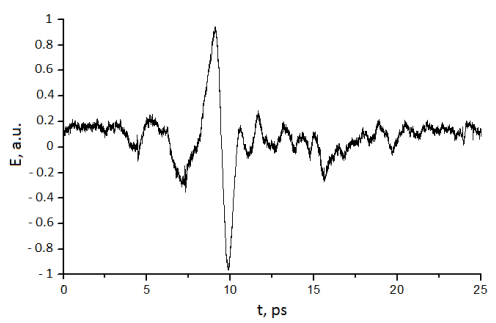

(c)

Figure 4. (a) THz pulse reflection from steel surface coated with epoxy glue Poxipol, (b) THz pulse reflection from steel surface coated with glue BF-6 in area without defect (c) in an area with defect.

Figure. 4 (b) shows the reflection of $\mathrm{THz}$ radiation from the steel sample coated with glue $\mathrm{BF}-6$ in the area without defect. One can see two reflected pulses from each surface similarly to the sample with glue Poxipol. The reflection from the same sample but from the area with defect is presented on Fig. 4 (c). There is no reflection from the glue-steel surface observed, and therefore the defect of the coating can be detected on the qualitative level. The real part of refraction index of the structure was extracted from the reference and sample spectra using the following equation: ${ }^{21}$

$$
n_{\mathrm{smpl}}(f)=1+\frac{c}{2 \pi f d}\left(\phi_{\mathrm{smpl}}(f)-\phi_{\mathrm{ref}}(f)\right),
$$

where $n_{\mathrm{smpl}}(f)$ is the real part of refractive index of the investigated structure, $c$ is the speed of light, $f$ is the frequency, $d$ is the sample thickness, $\phi_{\text {smpl }}$ and $\phi_{\text {ref }}$ are the phases of the complex amplitudes of the sample and the reference. The average refractive index of Poxipol glue $N=2,631$

In the second stage, we experimentally confirmed the possibility of thickness determination for the material with known refractive index. To create the sample with the varying thickness of the glue layer cruciform cut was slotted on the metal plate, which simulated defect. The depth of the cut was $0.5 \mathrm{~mm}$. Next, the plate with the defect was coated with glue Poxipol which was polished after drying. Fig. 5 shows sample before and after coating with the glue.

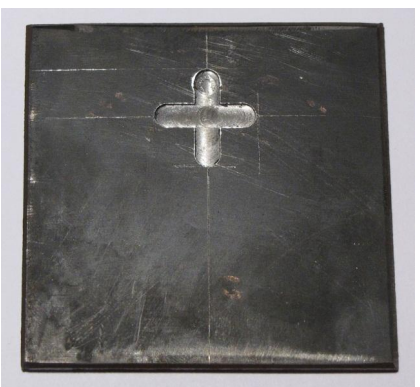

(a)

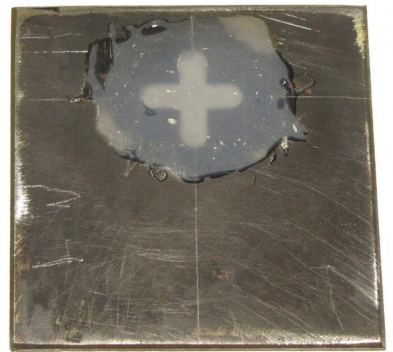

(b)

Figure 5. Sample for constructing a 3D thickness pattern (a) before coating with glue and (b) after.

Thickness measurement was carried out on area of $20 \times 20 \mathrm{~mm}^{2}$ by raster scanning with $2 \mathrm{~mm}$ increment. Reflection from this sample represents two pulses and delay between them linearly depends on thickness. Characteristic patterns of $\mathrm{THz}$ pulse reflected from cruciform cut area and area outside of it are shown on Fig. 6 (a) and (b), respectively.

Using formula (1) and knowing the refractive index of the glue we have obtained 3D model with layer thickness of Poxipol glue deposited on a metal substrate with a relief. 3D model is shown on Fig. 6 (c). For the pattern plotting it was assumed that the sample surface is flat and the thickness can be counted from it. 


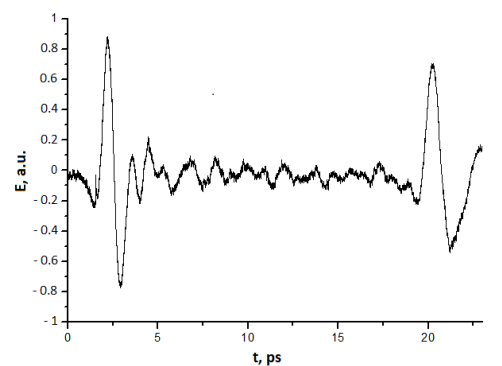

(a)

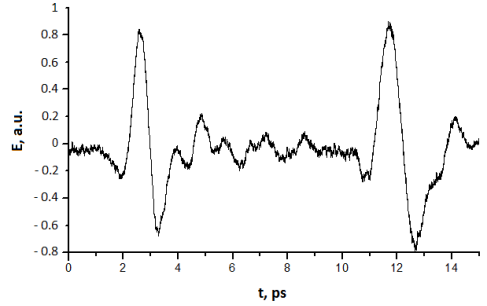

(b)

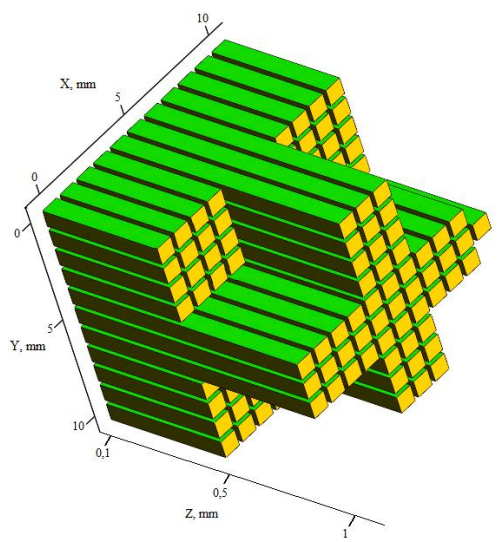

(c)

Figure 6. Characteristic patterns of $\mathrm{THz}$ pulse reflected from (a) cross area and (b) area outside the cross, (c) 3D thickness pattern of epoxy glue Poxipol coated on a relief steel plate.

\subsection{Delamination of composite material}

Next, we investigated the reflection of $\mathrm{THz}$ radiation from the region of the composite with the bundle structure. As the object was selected composite "1B" (Fig. 1c). Obtained time profiles were compared with the time profiles of the $\mathrm{THz}$ radiation reflected from the same composite in an area without bundles (Fig. 7).

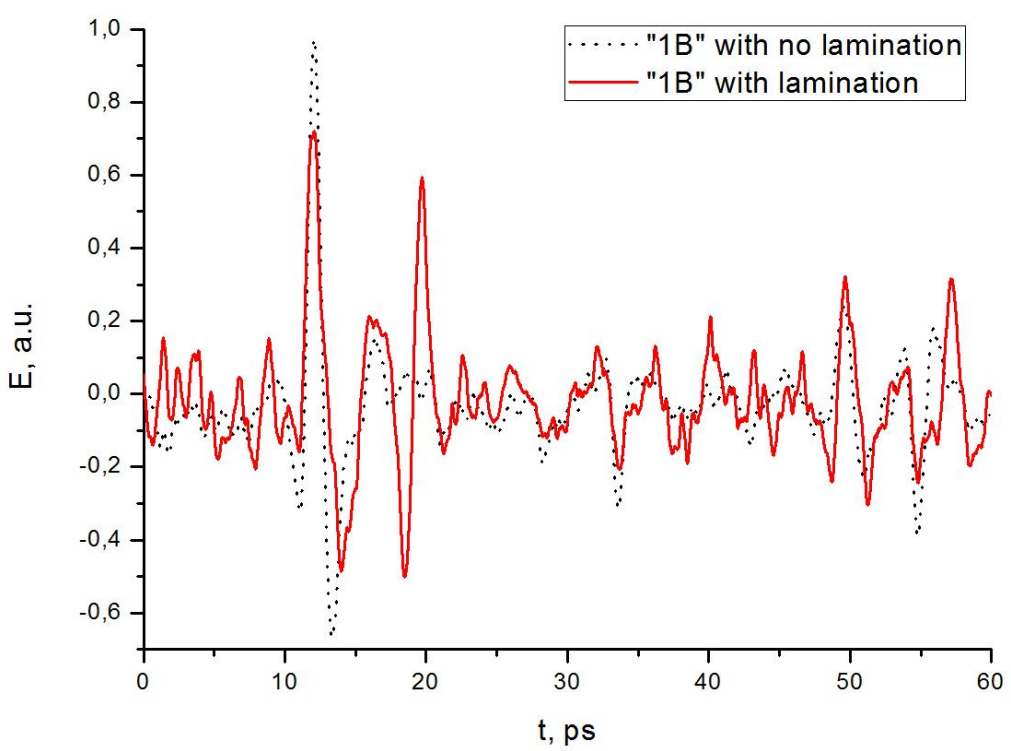

Figure 7. Temporal profiles of the $\mathrm{THz}$ radiation reflected from the area of the $1 \mathrm{~B}$ composite without bundle (dotted line) and with the bundle (solid line).

\subsection{Burn diagnostics using material property measurements}

Composite "16a" (Fig. 1a) was selected as the object of investigation. Time profiles of the THz pulse were obtained in the area, where the most visually noticeable difference.

It is noticeable that the amplitude of the $\mathrm{THz}$ pulse reflected from burned composite greater than the amplitude of $\mathrm{THz}$ pulse reflected from unburned (Fig. 8a). There are also changes in the spectrum at $0.56 \mathrm{THz}$, $0.6 \mathrm{THz}, 0.68 \mathrm{THz}$ (Fig. 8b). 


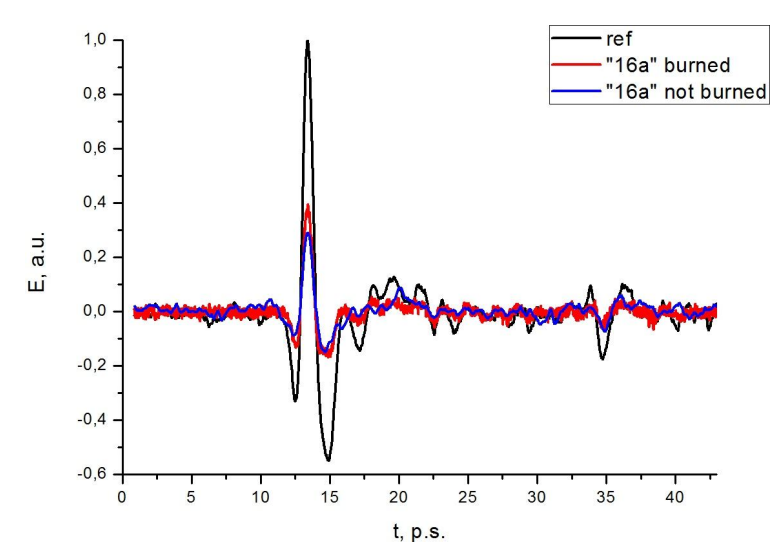

(a)

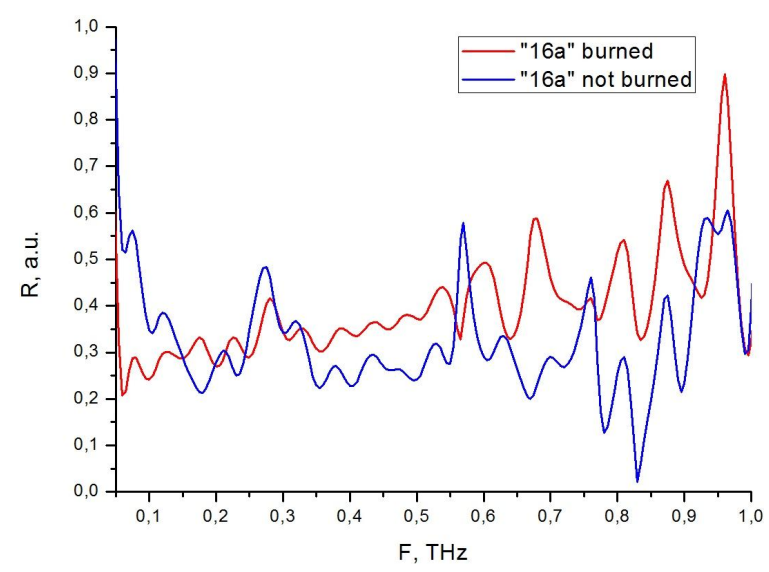

(b)

Figure 8. Comparison of $\mathrm{THz}$ (a) pulses and (b) spectra reflected from burned and unburned composites (b).

\subsection{2-D reflective imaging of stress defect}

The image of the sample (Fig. 1b) was obtained using the raster scanning THz TDS techniques. The wave forms of $\mathrm{THz}$ signal were measured for $40 \times 40$ points.

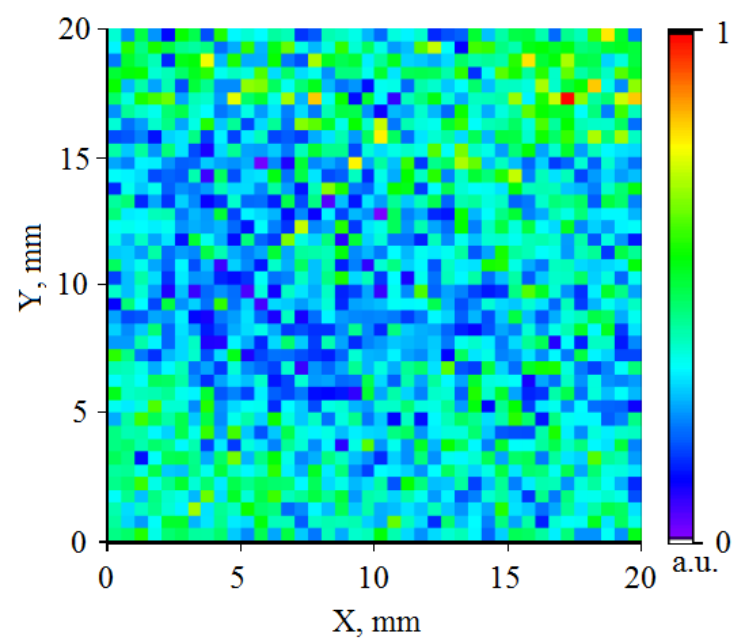

(a)

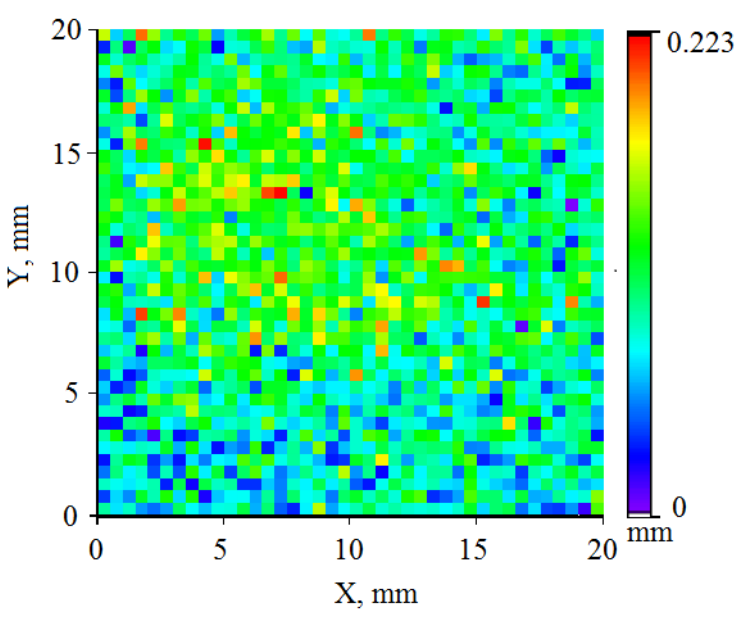

(b)

Figure 9. The spatial distribution of the amplitude (a) and phase (b) in the registration plane when summing in the range 0.01-0.09 THz

Fig. 9 shows the reconstructed mean amplitude and phase characteristics of the registered $\mathrm{THz}$ field in the range 0.01-0.09 THz passed through the defect. The summation of the amplitude reconstructions was carried by finding mean value for each pixel in a given range. The phase distribution was calculated as:

$$
h\left(x_{0}, y_{0}\right)=\frac{\sum_{i=1}^{m} \frac{\Delta \varphi\left(x_{0}, y_{0}\right) c}{2 \pi \nu_{i}\left(n\left(\nu_{i}\right)-1\right)}}{m},
$$

where $\Delta \varphi\left(x_{0}, y_{0}\right)$ is the frequency-domain unwrapped phase difference between reference and object fields at the object plane, $c$ - light velocity, $n\left(\nu_{i}\right)$ is the refractive index dispersion in a given range. Use of this formula assumes that the refractive index is constant in the whole area of the test sample and does not change by stress defect. However, the stress defect can change the refractive index of the composite in some areas. Therefore the calculation of numerical data about its relief is more complex and requires further investigation. 


\section{CONCLUSION}

THz TDS in reflection and transmission mode has several potential advantages over other nondestructive evaluation methods for inspection of aircraft glass fiber composites. Ultrasound technique require that the source and detector remain in contact with the aircraft. THz radiation can penetrate glass fiber without contacting it and detect surface defects, hidden voids and delaminations in composites.

An aircraft glass fiber composite with various forms of damage was examined using THz TDS system. Index of refraction and absorption coefficient in the terahertz frequency range were measured using THz TDS in transmission configuration for comparison of damaged and undamaged material states. Results showed that heat damage ( $843 \mathrm{~K}$ during 20 minutes) did not noticeably change the material properties of the composite sample.

\section{ACKNOWLEDGMENT}

This work was partially financially supported by Government of Russian Federation, Grant 074-U01.

Nikolay Petrov acknowledges financial support from Russian Ministry of Education and Science project within the state mission for institutions of higher education (Agreement No 2014/190).

\section{REFERENCES}

[1] Blitz, J. and Simpson, G., [Ultrasonic methods of non-destructive testing], Springer, London. (1996).

[2] Castaings, M., Cawley, P., Farlow, R., and Hayward, G., "Single sided inspection of composite materials using air coupled ultrasound," J. Nondestruct. Eval. 17(1), 37-45 (1998).

[3] Avdelidis, N. P., Almond, D. P., and Hawtin, B. C., "Transient thermography in the assessment of defects of aircraft composites," NDT \& E Int 36(6), 433-439 (2003).

[4] Hung, Y., Chen, Y., Ng, S., Liu, L., Huang, Y., Luk, B., Ip, R., Wu, C., and Chung, P., "Review and comparison of shearography and active thermography for nondestructive evaluation," Mater. Sci. Eng.: R: Rep. 64(5-6), 73 - 112 (2009).

[5] Almond, D. P. and Peng, W., "Thermal imaging of composites," J. Microsc. 201(2), 163-170 (2001).

[6] Hung, Y., "Applications of digital shearography for testing of composite structures," Compos. Part BEng 30(7), 765 - 773 (1999).

[7] Katnam, K., Silva, L. D., and Young, T., "Bonded repair of composite aircraft structures: A review of scientific challenges and opportunities," Prog. Aerosp. Sci. 61(0), $26-42$ (2013).

[8] Shen, Y. C., Taday, P. F., and Pepper, M., "Elimination of scattering effects in spectral measurement of granulated materials using terahertz pulsed spectroscopy," Appl. Phys. Lett. 92(5), 051103-051103-3 (2008).

[9] Fletcher, J. R., Swift, G. P., Dai, D. C., Levitt, J. A., and Chamberlain, J. M., "Propagation of terahertz radiation through random structures: An alternative theoretical approach and experimental validation," $J$. Appl. Phys. 101(1), - (2007).

[10] Wietzke, S., Jansen, C., Rutz, F., Mittleman, D., and Koch, M., "Determination of additive content in polymeric compounds with terahertz time-domain spectroscopy," Polym. Test. 26(5), $614-618$ (2007).

[11] Stoik, C. D., Bohn, M. J., and Blackshire, J. L., "Nondestructive evaluation of aircraft composites using transmissive terahertz time domain spectroscopy," Opt. Express 16(21), 17039-17051 (2008).

[12] Petrov, N. V., Gorodetsky, A. A., and Bespalov, V. G., "Holography and phase retrieval in terahertz imaging," Proc. SPIE 8846, 88460S-88460S-7 (2013).

[13] Petrov, N. V., Bespalov, V. G., and Volkov, M. V., "Phase retrieval of thz radiation using set of 2d spatial intensity measurements with different wavelengths," Proc. SPIE 8281, 82810J-82810J-7 (2012).

[14] Petrov, N. V., Galiaskarov, A. N., Nikolaeva, T. Y., and Bespalov, V. G., "The features of optimization of a phase retrieval technique in THz frequency range," Proc. SPIE 8413, 84131T-84131T-5 (2012).

[15] Gürtler, A., Winnewisser, C., Helm, H., and Jepsen, P. U., "Terahertz pulse propagation in the near field and the far field," J. Opt. Soc. Am. A 17(1), 74-83 (2000).

[16] Izumida, S., Ono, S., Liu, Z., Ohtake, H., and Sarukura, N., "Spectrum control of thz radiation from inas in a magnetic field by duration and frequency chirp of the excitation pulses," Appl. Phys. Lett. 75(4), 451-453 (1999). 
[17] Mittleman, D. M., Cunningham, J., Nuss, M. C., and Geva, M., "Noncontact semiconductor wafer characterization with the terahertz hall effect," Appl. Phys. Lett. 71(1), 16-18 (1997).

[18] Lee, Y.-S., [Principles of terahertz science and technology], Springer Science+Business Media, New York. (2009).

[19] Chan, W. L., Deibel, J., and Mittleman, D. M., "Imaging with terahertz radiation," Rep. Prog. Phys. 70(8), 1325 (2007).

[20] Grischkowsky, D., Keiding, S., van Exter, M., and Fattinger, C., "Far-infrared time-domain spectroscopy with terahertz beams of dielectrics and semiconductors," J. Opt. Soc. Am. B 7, 2006-2015 (Oct 1990).

[21] Zhang, X.-C. and Xu, J., [Introduction to THz Wave Photonics], Springer, New York. (2010). 